\section{Esophageal Cancer: The Paradigm for Teamwork}

The March issue of JNCCN detailed moving from the "good to the perfect" in managing Hodgkin disease, one of the more treatable and curable cancers. The articles in this issue, such as the one by Das et al. (Combined modality therapy of localized gastric and esophageal cancer), vividly highlight the painstaking steps involved in making headway against some of the more resistant tumors, such as esophageal tumors. The encouraging news is that the oncology disciplines are coming together in their attack on the disease. Surgical, radiation, and medical oncology are collaborating in designing new strategies and are submitting these strategies to the scrutiny of laborious clinical trials. Progress, albeit slow, is being made.

A major step appears to be confirmation that combined modality therapy can lead to pathological complete response, which appears to translate into a survival advantage. Thus, in a recent analysis, the $22 \%$ of patients undergoing chemoradiation who experienced complete response at esophagectomy had a 3-year survival rate of $64 \%$, compared with $34 \%$ for patients with residual tumor. ${ }^{1}$

And yet, we are really just beginning. For example, as the Das et al. article points out, "Our inability to individualize therapy for localized upper gastrointestinal cancer patients remains a major setback." Are types I, II, and III tumors of the gastroesophageal region the same or does each require a different approach? Do squamous and adenocarcinomas behave the same? Are the treatment strategies for stage II disease the same as should be used in stage IVa disease? Furthermore, each of these questions requires a rigorous trial design to illustrate what may be subtle nuances in optimal disease management.

Ultimately, of course, it is the interjection of new therapies, new combinations, and new schedules that may lead to significant incremental changes. Whether new doublets or triplets will produce synergies to improve effectiveness can only be tested through methodical exploration. The discovery of potential new combinations can be hastened by judicious use of randomized phase II studies and then moving the leading candidate regimens into definitive testing. Similarly, novel timing and scheduling must be explored. Determinations such as neoadjuvant versus postoperative approaches and combined versus sequential therapies must be made. And, hopefully, the myriad of new targeted therapies will add substantially to the armamentarium.

This article illustrates another concern that must be addressed before optimal results are achieved: not only must the right step be taken, it must be taken in the right way. Multimodality chemoradiation and the technical complexity of esophageal surgery make measures to ensure that care is delivered by sophisticated teams with the requisite skills and resources to meet the extraordinary demands of these procedures a necessary paradigm. The public is vitally interested in this activity, and groups such as Leapfrog have proposed volume standards of more than 13 cases per year for performing esophagectomy. ${ }^{2}$ Similarly, radiation therapy planning must also be of the highest caliber to deliver appropriate doses while shielding vital structures.

The path is clear: a 2-pronged approach is needed. First, new approaches must be systematically investigated, each trial building on the one before, so that new patterns and innovative approaches emerge and are incorporated as standards. Second, assuring that only the best techniques are used and outcomes are of uniform high quality must be continually emphasized. The mechanism for reaching these goals is the same: formal teamwork and a collaborative orientation. Through these collegial efforts, esophageal cancer will eventually be added to the list of more manageable tumors.

\section{References}

1. Donington JS, Miller DL, Allen MS, et al. Tumor response to induction chemoradiation: influence on survival after esophagectomy. Eur J Cardiothorac Surg 2003;24:631-636.

2. Leapfrog Group for Patient Safety. Fact sheet. Available at www.leapfroggroup.org/media/file/ Leapfrog-Evidence-based_Hospital_Referral_Fact_Sheet.pdf. Last accessed March 10, 2006.

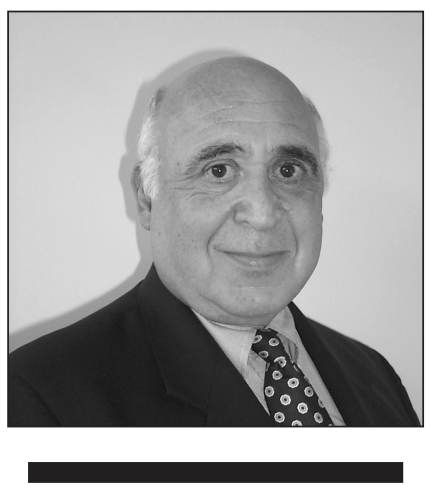

By Rodger J. Winn, MD

Rodger J. Winn is the Editorin-Chief of JNCCN. He is Clinical Consultant at the National Quality Forum, and his past positions include Associate Professor of Clinical Medicine at The University of Texas M. D.

Anderson Cancer Center. Dr. Winn received his medical degree from Jefferson Medical College of Philadelphia. His postgraduate training includes an internship and residency at Jefferson Medical College, and he also completed a medical oncology fellowship at Memorial Sloan-Kettering Cancer Center in New York. He is board certified in internal medicine and holds subspecialty certification in oncology. 